\title{
Comparative Transcriptome Analysis Identifies Candidate Genes Related to Black-Spotted Pattern Formation in Spotted Scat (Scatophagus argus)
}

\author{
Xiaozhan Lin ${ }^{1,2,+}$, Changxu Tian ${ }^{1,2,3,+} \mathbb{D}$, Yang Huang ${ }^{1,2,3}$, Hongjuan Shi ${ }^{1,2,3}$ and Guangli Li $1,2,3, *(\mathbb{D})$ \\ 1 Fisheries College, Guangdong Ocean University, Zhanjiang 524088, China; \\ linxiaozhan@stu.gdou.edu.cn (X.L.); tiancx@gdou.edu.cn (C.T.); zjouhy@126.com (Y.H.); \\ shihongjuan1990@163.com (H.S.) \\ 2 Guangdong Research Center on Reproductive Control and Breeding Technology of Indigenous Valuable Fish \\ Species, Guangdong Provincial Engineering Laboratory for Mariculture Organism Breeding, Guangdong \\ Provincial Key Laboratory of Pathogenic Biology and Epidemiology for Aquatic Economic Animals, \\ Guangdong Ocean University, Zhanjiang 524088, China \\ 3 Southern Marine Science and Engineering Guangdong Laboratory (Zhanjiang), Zhanjiang 524088, China \\ * Correspondence: ligl@gdou.edu.cn; Tel.: +86-759-2383124; Fax: +86-759-2382459 \\ + These authors contributed equally to this work.
}

Citation: Lin, X.; Tian, C.; Huang, Y.; Shi, H.; Li, G. Comparative Transcriptome Analysis Identifies Candidate Genes Related to Black-Spotted Pattern Formation in Spotted Scat (Scatophagus argus). Animals 2021, 11, 765. https:// doi.org/10.3390/ani11030765

Academic Editor: Kenji Saitoh

Received: 25 January 2021

Accepted: 7 March 2021

Published: 10 March 2021

Publisher's Note: MDPI stays neutral with regard to jurisdictional claims in published maps and institutional affiliations.

Copyright: (c) 2021 by the authors. Licensee MDPI, Basel, Switzerland. This article is an open access article distributed under the terms and conditions of the Creative Commons Attribution (CC BY) license (https:// creativecommons.org/licenses/by/ $4.0 /)$.
Simple Summary: Spotted scat (Scatophagus argus) is a commercially important marine aquaculture and ornamental fish species in China and East Asian countries. There are dozens of black spots on each side of the body, and the caudal fin, which is yellow and black, is appreciated in ornamental fish markets. To explore the genetic mechanisms of its pattern formation, we found 2357 differentially expressed genes (DEGs) by comparing the transcriptome in the black-spotted skin, non-spotted skin and caudal fin in S. argus. The results will expand our knowledge about the molecular mechanism of important genes and pathways associated with pigment pattern formation and provide a certain theoretical basis for the molecular breeding in S. argus.

Abstract: Spotted scat (Scatophagus argus) is an economically important marine aquaculture and ornamental fish species in Asia, especially in southeast China. In this study, skin transcriptomes of S. argus were obtained for three types of skin, including black-spotted skin (A), non-spotted skin (B) and caudal fin (C). A total of nine complementary DNA (cDNA) libraries were obtained by Illumina sequencing. Bioinformatics analysis revealed that 1358, 2086 and 487 genes were differentially expressed between $\mathrm{A}$ and $\mathrm{B}, \mathrm{A}$ and $\mathrm{C}$, and $\mathrm{B}$ and $\mathrm{C}$, respectively. The results revealed that there were 134 common significantly differentially expressed genes (DEGs) and several key genes related to pigment synthesis and pigmentation, including tyrp1, mitf, pmel, slc7a2, tjp1, hsp70 and mart-1. Of these, some DEGs were associated with pigmentation-related Kyoto Encyclopedia of Genes and Genomes (KEGG) pathways, such as tyrosine metabolism, melanogenesis, the Wnt signaling pathway and the mitogen-activated protein kinase (MAPK) signaling pathway. The results will facilitate understanding the molecular mechanisms of skin pigmentation differentiation in S. argus and provide valuable information for skin coloration, especially the formation of spotted patterns on other marine fish species.

Keywords: Illumina sequencing; pigment synthesis; molecular mechanisms; skin coloration

\section{Introduction}

Skin pigmentation and color patterns are the most diverse phenotypic characteristics in animals. Their functions are closely related to adaptation to different environments, including temperature regulation, camouflage, social interaction, spouse choice, etc. [1-3]. The formation of color patterns is determined by various pigments synthesized by chromophores or pigment cells. The color of pigment cells and pigment pattern formation 
and maintenance are related to the nutritional, physiological, genetic and environmental factors, making pigmentation a complex biological process [4]. Of these, the genetic basis is the most important factor affecting skin pigmentation through gene regulation. At present, genetic and genomic analysis techniques are used to explore the mechanisms of skin pigmentation.

Six types of pigment cells have been reported in vertebrates. Among them, only melanocytes are found in the skin of mammals, and only teleost fishes have six types of pigment cells, including melanophores (black, dark brown), xanthophores (yellow), erythrophores (red/orange), iridophores (reflecting), leucophores (white) and cyanophores (blue) [5-8]. These chromatophores synthesize and retain their specific pigments and inner structures intracellularly [9]. Skin pigmentation and pattern reflect the numbers and arrangements of the chromatophores. Melanophores and other types of pigment cells are also focused on pattern formation. Melanophores and melanin are the main factors that determine skin color. The molecular melanin biosynthesis mechanism has been widely studied in vertebrates. The melanogenesis pathway is conservative $[10,11]$. Melanin synthesis takes place within the melanosomes of melanocytes and involves the tyrosine metabolism pathway. Tyrosinases (TYRs), tyrosinase-related protein 1 (TYRP1) and dopachrome tautomerase (DCT) are important enzymes in melanin synthesis [12,13]. The increased expression of the microphthalmia-associated transcription factor (MITF) and its activation by phosphorylation stimulate the transcription of TYR, TYRP1 and DCT [12,14]. However, the biosynthesis of other types of pigments via different pathways and regulatory networks is less studied [6]. Several reports were recorded about pigmentation on model organisms such as medaka (Oryzias latipes) and zebrafish (Danio rerio) $[15,16]$.

Spotted scat (Scatophagus argus) is a kind of edible and ornamental fish. It is a commercially important marine species in China and East Asian countries owing to its high nutrition and delicate flavor. There are dozens of black spots on both sides of the fish body, and the caudal fin is yellow and black. The molecular mechanisms underlying the formation of the body color, especially the black spots in S. argus, are not well understood. We used RNA-Seq to detect differentially expressed genes (DEGs) by comparing the blackspotted skin, non-spotted skin and caudal fin in S. argus. The study aimed to detect the important genes and pathways associated with black-spotted pigment pattern formation in S. argus.

\section{Materials and Methods}

\subsection{Ethical Statements}

This study was approved by the Institutional Animal Care and Use Committee (IACUS) of Guangdong Ocean University (Zhanjiang, China). Sampling procedures complied with the guidelines of the IACUS on the care and use of animals for scientific purposes.

\subsection{Sample Preparation}

The experimental fishes (S. argus) (Figure 1, total length: $17.27 \pm 1.52 \mathrm{~cm}$; body weight: $147.90 \pm 14.62 \mathrm{~g}$ ) were obtained from Donghai Island, Guangdong, China, and were kept in an outdoor cement pool with water (water temperature: $25^{\circ} \mathrm{C}$ ) for one week. Black-spotted skin (A), non-spotted skin (B) and caudal fin (C, contained both black and yellow areas) tissues $\left(0.8-1 \mathrm{~cm}^{2}, 40-50 \mathrm{mg}\right)$ were collected and stored in liquid nitrogen for RNA isolation. The same tissues of every three fishes were used as a sample group, forming a total of nine libraries. 


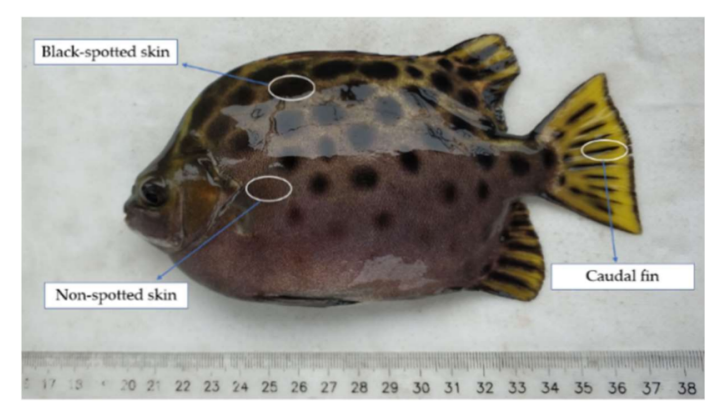

Figure 1. Sampling distribution of different color parts of S. argus (black-spotted skin; nonspotted skin; caudal fin).

\subsection{RNA Extraction and Illumina Library Preparation}

The total RNA ( $n=3$ per group) from the three groups was extracted using TRIzol reagent (Life Technologies, Carlsbad, CA, USA) following the manufacturer's instructions. The RNA purity (Table S1) was monitored by Nano spectrophotometer (Nanodrop 2000c, Thermo Scientific, Wilmington, DE, USA). The RNA integrity was checked by ethidium bromide staining of $28 \mathrm{~S}$ and $18 \mathrm{~S}$ ribosomal bands on a $1.0 \%$ agarose gel. Then, a total of $3 \mu \mathrm{g}$ of RNA was prepared for each Illumina library sequence. Sequencing libraries were generated using the NEBNext ${ }^{\circledR}$ Ultra ${ }^{\mathrm{TM}}$ RNA Library Prep Kit for Illumina ${ }^{\circledR}$ (NEB, New England Biolabs, Palo Alto, CA, USA) following the manufacturer's instructions. The library fragments were purified using the AMPure XP system (Beckman Coulter, Beverly, MA, USA) to select 250-300 bp complementary DNA (cDNA) fragments, and each library quality was assessed by the Agilent Bioanalyzer 2100 system (Agilent Technologies, Palo Alto, CA, USA). Then, $3 \mu \mathrm{L}$ of USER Enzyme (NEB, New England Biolabs, Palo Alto, CA, USA) was used with size-selected, adaptor-ligated cDNA at $37^{\circ} \mathrm{C}$ for $15 \mathrm{~min}$, followed by 5 min at $95{ }^{\circ} \mathrm{C}$ before PCR. PCR was performed with Phusion High-Fidelity DNA polymerase, universal PCR primers and Index $(X)$ Primer. Finally, PCR products were purified by the AMPure XP system and the library quality was assessed by the Agilent Bioanalyzer 2100 system. All clean libraries of sequencing data were submitted to the NCBI Sequence Read Archive (SRA) database (Accession No.: PRJNA670377).

\subsection{Data Filtering, Reads Mapping and Differential Expression Analysis}

Based on the $S$. argus reference genome and gene model annotation files (unpublished data), an index of the reference genome was built, and the paired-end clean reads were aligned to the reference genome using Hisat2 v2.0.5 (https:/ / anaconda.org/biobuilds/ hisat2) (accessed on 16 August 2020). The gene expression levels were estimated by fragments per kilobase of exon model per million reads mapped (FPKM) [17]. Clean reads were picked out by removing reads containing adapter, poly-N and low-quality reads from raw data, which were processed through in-house perl scripts. DESeq2 R package (version 1.16.1) was used to identify DEGs [18] with a threshold of $\mid \log _{2}$ fold change $\mid \geq 2.0$ and $p$-value (padj) $<0.05$ [19]. The DEGs were mapped to the Kyoto Encyclopedia of Genes and Genomes (KEGG) (http:/ / www.genome.jp/kegg/)) (accessed on 22 September 2020) and Gene Ontology $(\mathrm{GO})$ databases (padj $\leq 0.05$ ).

\subsection{Validation of DEGs by Quantitative Real-Time Polymerase Chain Reaction (qRT-PCR)}

A total of 14 DEGs were randomly selected from black-spotted skin vs. non-spotted skin, black-spotted skin vs. caudal fin, and non-spotted skin vs. caudal fin to verify the expression of DEGs. The primers of the selected genes were designed using Primer Premier software v5.0, which are listed in Table S2. qRT-PCR was performed using SYBR Green qPCR Mix (Dongsheng Biotech, Guangzhou, China) on a Light Cycler real-time quantitative PCR system (Roche, Basel, Switzerland) according to the manufacturer's instructions. $\beta$-actin gene was used as a reference in order to standardize the gene expression values. PCR amplification was performed in triplicate for each gene. Relative expression levels 
were measured in terms of the threshold cycle value and were normalized using the $2^{-\Delta \Delta C t}$ method [20].

\section{Results}

\subsection{Raw Sequencing Reads and Quality Statistics}

Nine cDNA libraries were built from three different body parts of the fish. In Table 1, we determined the raw reads, clean reads, clean bases, Q30 percentages and GC percentages in order to describe the quality of the transcriptomic data from S. argus. A total of 485.6 million raw reads were generated after removing the reads, containing adapter, ploy-N reads and the lower quality reads from raw data. We got 478.1 million clean reads, and the percentages of Q30 bases were more than $92 \%$ for all the samples, indicating a high-quality sequence.

Table 1. Summary of the transcriptomic data from S. argus.

\begin{tabular}{|c|c|c|c|c|c|}
\hline Group & Raw Reads & Clean Reads & Clean Bases (G) & Q30 (\%) & GC Content (\%) \\
\hline \multicolumn{6}{|c|}{$\begin{array}{l}\text { Black-spotted skin } \\
\text { (A) }\end{array}$} \\
\hline A1 & $58,647,296$ & $57,844,474$ & 8.68 & 93.16 & 46.97 \\
\hline $\mathrm{A} 2$ & $54,843,424$ & $53,929,484$ & 8.09 & 92.84 & 46.67 \\
\hline A3 & $61,189,662$ & $60,096,498$ & 9.01 & 94.12 & 46.56 \\
\hline \multicolumn{6}{|c|}{$\begin{array}{l}\text { Non-spotted skin } \\
\text { (B) }\end{array}$} \\
\hline B1 & $55,148,934$ & $54,250,656$ & 8.14 & 92.72 & 45.94 \\
\hline B2 & $53,000,358$ & $52,199,276$ & 7.83 & 92.69 & 46.70 \\
\hline B3 & $56,077,298$ & $55,093,088$ & 8.26 & 92.64 & 46.07 \\
\hline \multicolumn{6}{|c|}{ Caudal fin (C) } \\
\hline $\mathrm{C} 1$ & $46,402,098$ & $45,867,418$ & 6.88 & 92.16 & 46.15 \\
\hline $\mathrm{C} 2$ & $50,405,758$ & $49,913,528$ & 7.49 & 92.47 & 46.31 \\
\hline $\mathrm{C} 3$ & $49,958,956$ & $48,909,024$ & 7.34 & 94.57 & 46.88 \\
\hline
\end{tabular}

\subsection{Comparative Analysis of DEGs}

The results showed that compared with the black-spotted skin, 1358 genes were differentially expressed in the non-spotted skin. Of these, 1343 genes were upregulated and 15 genes were downregulated. In the caudal fin, a total of 2086 genes were differentially expressed, with 2019 genes being upregulated and 67 genes being downregulated. Compared with the caudal fin, 487 genes were differentially expressed in the non-spotted skin. Of these, 90 genes were upregulated and 397 genes were downregulated (Figure 2a). In addition, a total of 134 coexpressed genes showed significant differential expression levels in three different groups (Figure $2 \mathrm{~b}$ ), which may be related to skin coloration and skin derivatives production in $S$. argus.

\subsection{GO and KEGG Pathway Enrichment Analysis}

We made a comparison among the A (black-spotted skin), B (non-spotted skin) and C (caudal fin) transcriptomes to explore whether the mechanism underlying the black-spotted skin color formation of $S$. argus was controlled by multiple agents via pathways. DEGs identified in three different comparisons were used for the GO and KEGG functional enrichment analysis. According to the GO functional analysis, DEGs were classified into three major functional categories, including biological process (BP), cellular component (CC) and molecular function (MF). In each comparison, the top ten most significant enrichment pathways were selected in three different categories. The detailed annotations for each category are depicted in Figure $3 \mathrm{a}-\mathrm{c}$. We found that monovalent inorganic cation transport (GO:0015672), ion transport (GO:0006811), pyruvate metabolic process (GO:0006090) and glycolytic process (GO:0006096) were the most enriched components in the BP category; troponin complex (GO:0005861), striated muscle thin filament (GO:0005865), cytoskeletal 
part (GO:0044430) and supramolecular complex (GO:0099080) were the enriched terms in the CC category; ion transmembrane transporter activity (GO:0015075) and ion channel activity (GO:0005216) were the most common terms in the MF category.
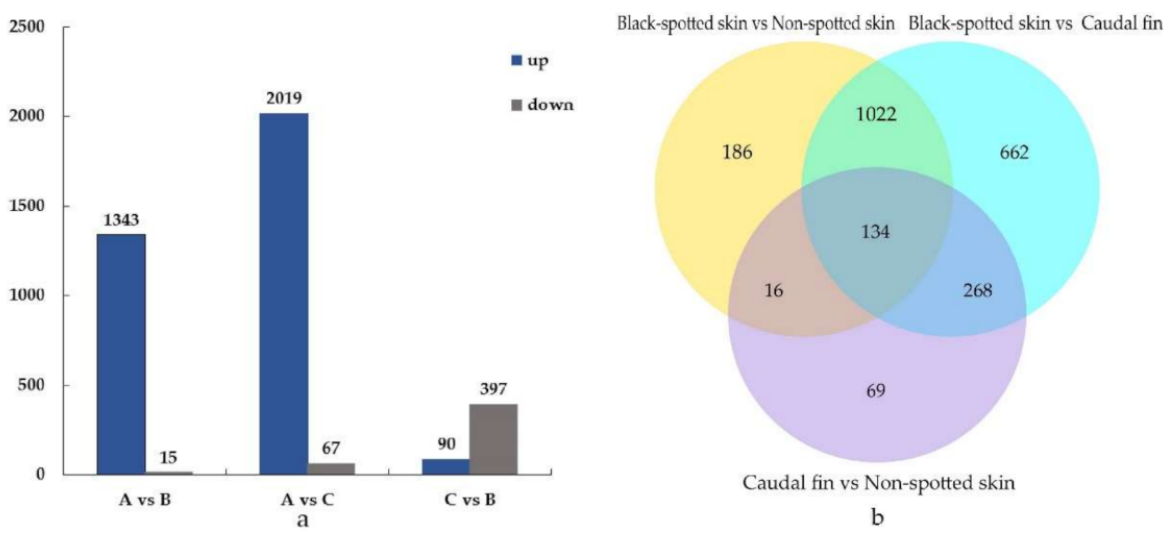

b

Figure 2. (a) Comparison of the number of differentially expressed genes (DEGs) in three different tissues of $S$. argus. The horizontal axis represents the samples on the control of the situation, and the vertical axis measures the difference of gene numbers. There are two expression levels. The blue color stands for the upregulated expression, and the gray color represents the downregulated expression. (b) DEG number and Venn diagram of the overlap of the different groups. A (black-spotted skin), B (non-spotted skin) and C (caudal fin).
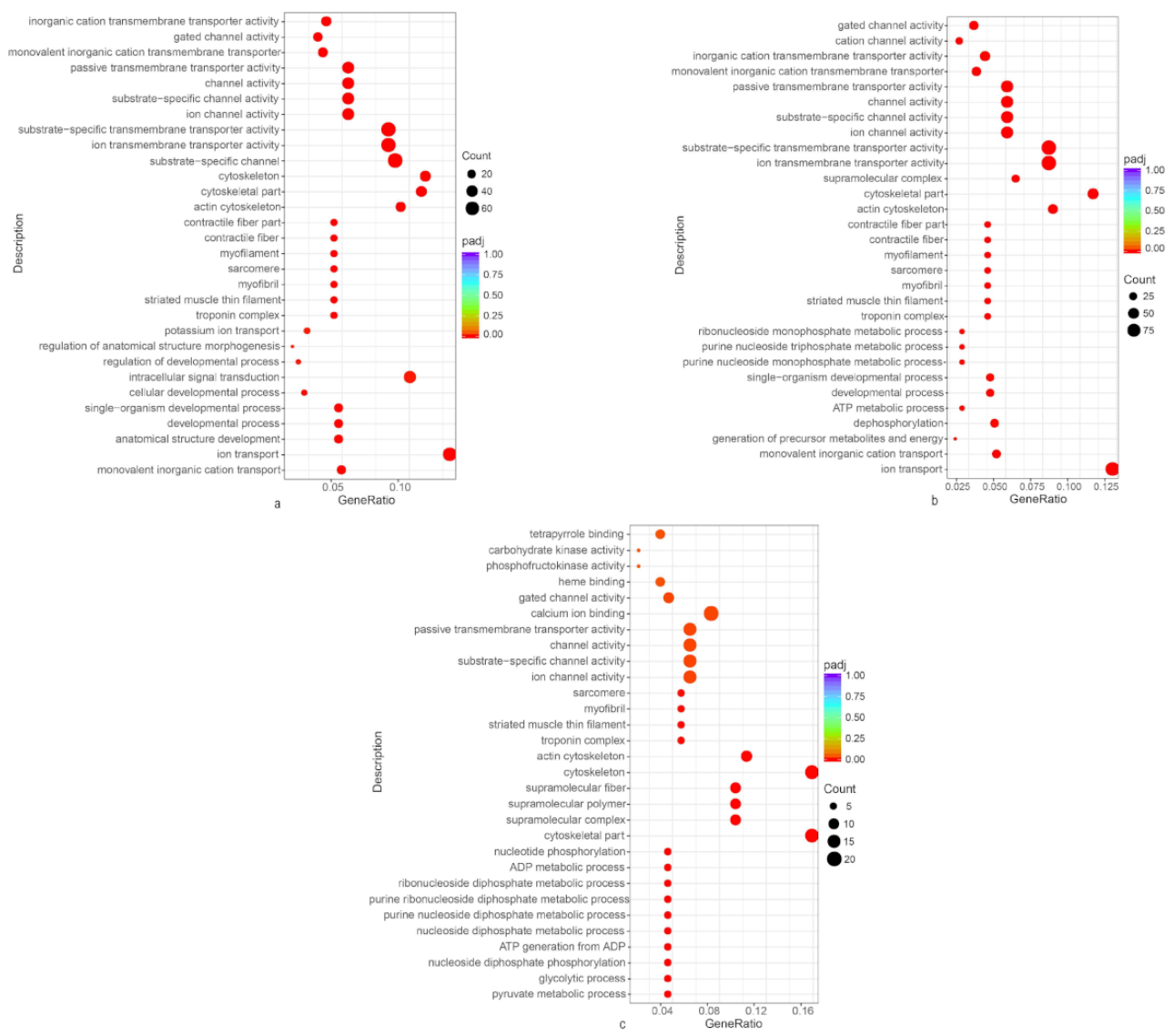

Figure 3. Gene Ontology (GO) function classification of the differentially expressed genes (DEGs) comparison between the groups (padj < 0.05). (a) Black-spotted skin vs. non-spotted skin; (b) Blackspotted skin vs. Caudal fin; (c) Caudal fin vs. non-spotted skin. The x-axis represents the number of genes, and the y-axis represents GO terms. 
A total of 297 KEGG pathways were found in the study. The top 10 pathways in each comparison were selected, as shown in Table S3. The pathways with most of the represented genes were the oxidative phosphorylation, cardiac muscle contraction, focal adhesion, neuroactive ligand-receptor interaction and calcium signaling pathways. As shown in Table 2, some DEGs were related to pigmentation. We mainly focused on genes related to melanin synthesis, deposition and migration. The KEGG pathway analysis results showed that some DEGs were associated with pigmentation-related pathways, such as tyrosine metabolism, melanogenesis, the Wnt signaling pathway and the MAPK signaling pathway.

Table 2. Analysis of differentially expressed genes (DEGs) related to pigmentation.

\begin{tabular}{|c|c|c|c|}
\hline Gene & Gene ID & Note & $\log _{2}$ Fold Change \\
\hline \multicolumn{4}{|c|}{ Black-spotted skin vs. Non-spotted skin } \\
\hline Tyrp1 & EVM0000142 & Tyrosinase-related protein 1 & 2.68 \\
\hline Wht2 & EVM0010869 & Wingless-type MMTV integration site family, member 2 & 8.04 \\
\hline Camk2 & EVM0018979 & Calcium/calmodulin-dependent protein kinase & 2.75 \\
\hline Adcy5 & EVM0008013 & Adenylate cyclase 5 & 2.12 \\
\hline Adcy6 & EVM0004537 & Adenylate cyclase 6 & 2.76 \\
\hline Wnt11 & EVM0010741 & Wingless-type MMTV integration site family, member 11 & 5.96 \\
\hline Foxd & EVM0009699 & Forkhead box protein D & 4.67 \\
\hline Mapk4 & EVM0001218 & Mitogen-activated protein kinase 4 & 3.51 \\
\hline Adcy9 & EVM0003371 & Adenylate cyclase 9 & 2.29 \\
\hline$H g f$ & EVM0021385 & Hepatocyte growth factor & 2.29 \\
\hline $\operatorname{Tgfb3}$ & EVM0004513 & Transforming growth factor beta- 3 & 2.96 \\
\hline Rasgrf1 & EVM0005858 & Ras-specific guanine nucleotide-releasing factor 1 & 4.77 \\
\hline Efna & EVM0012046 & Ephrin-A & -3.81 \\
\hline Mart-1 & EVM0011519 & Melanoma antigen recognized by T-cells 1 & 2.45 \\
\hline Tjp1 & EVM0021943 & Tight junction protein 1 & 2.12 \\
\hline Hsp70 & EVM0013804 & Heat shock $70 \mathrm{kDa}$ protein $12 \mathrm{~A}$ isoform $\mathrm{X} 1$ & 2.39 \\
\hline \multicolumn{4}{|c|}{ Black-spotted skin vs. Caudal fin } \\
\hline Pmel & EVM0007408 & Premelanosome protein & 2.23 \\
\hline Slc7a2 & EVM0021987 & Solute carrier family 7 member 2 & 4.29 \\
\hline $\operatorname{Pax} 3$ & EVM0000393 & Paired box protein 3 & 2.45 \\
\hline Pdgfrb & EVM0022774 & Platelet-derived growth factor receptor beta & 3.89 \\
\hline Flt1 & EVM0007297 & FMS-like tyrosine kinase 1 & 3.04 \\
\hline Dusp & EVM0020294 & Dual specificity MAP kinase phosphatase & 2.04 \\
\hline Hspb1 & EVM0002084 & Heat shock protein beta-1 & 4.37 \\
\hline$K d r$ & EVM0020836 & Kinase insert domain protein receptor & 2.96 \\
\hline Ntrk2 & EVM0017245 & Neurotrophic tyrosine kinase receptor type 2 & 4.74 \\
\hline
\end{tabular}

\subsection{Validation of Gene Expression Levels}

A total of 14 DEGs were selected and analyzed using qRT-PCR (Figure 4). The changing trends of those genes from qRT-PCR were similar to the results from the RNA-seq expression analysis, which supports the reliability of the transcriptome data. 


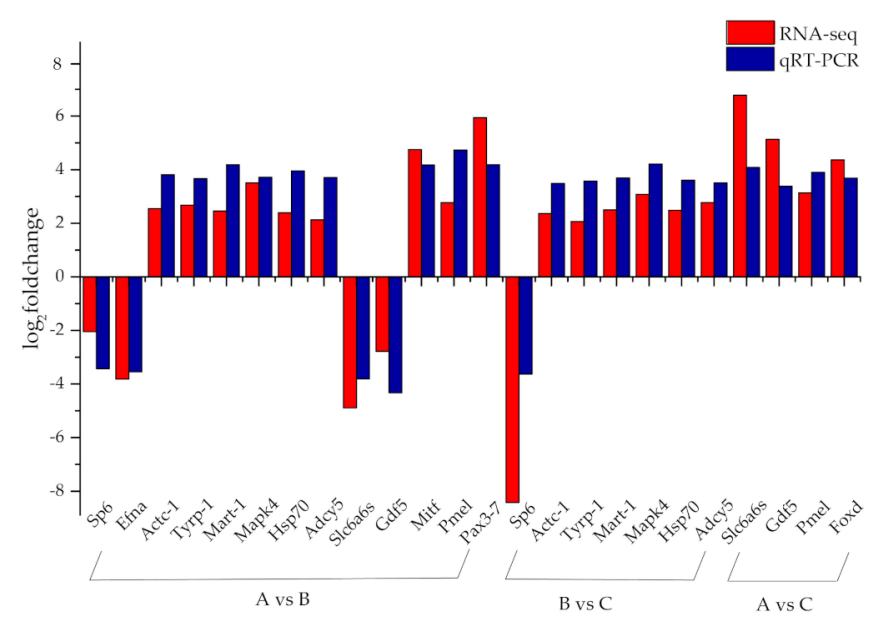

Figure 4. Comparison of gene expression data between RNA-seq and quantitative real-time polymerase chain reaction (PCR) (qRT-PCR). The $x$-axis presents the gene name, and the $y$-axis presents a fold change in gene expression.

\section{Discussion}

Different mechanisms are responsible for the changes in skin pigment patterns in animals. Black spot pigment pattern is the most important phenotypic feature of $S$. argus. However, the mechanism of formation is still unknown. We conducted a comparative transcriptome analysis of three differently pigmented regions of the adult black-spotted skin, non-spotted skin and caudal fin to screen for genes potentially involved in the maintenance of a specific pigment pattern in S. argus. The results showed that more differentially expressed genes were upregulated in black-spotted skin when compared to non-spotted skin and caudal fin, indicating that the formation process of black spots on the skin was complex and that more genes needed to participate in the process. As shown in Figure 2, there were DEGs and 134 shared coexpressed genes in three comparisons. In addition, the top 30 functional GO terms and top 10 KEGG pathways were enriched in three groups (Figure 3 and Table S3). The KEGG pathway analysis showed that a substantial number of DEGs between the three different regions were associated with pigmentation-related pathways (e.g., MAPK signaling pathway, Wnt signaling pathway, tyrosine metabolism) and cell-cell communication, such as focal adhesion, the calcium signaling pathway and ECM-receptor interaction.

In this study, differential expressions were observed by comparing differently colored regions in S. argus, most probably associated with specific expression profiles of individual pigment cell types in a particularly colored skin region [21]. The two most common types of melanin are eumelanin and pheomelanin in melanophore. Eumelanin corresponds to a brown/black color, while pheomelanin corresponds to a red/yellow color. The expression levels of eumelanin-related genes (such as tyrp1, mart-1, hsp70) in black-spotted skin were significantly higher than those in non-spotted skin. After investigating the DEGs from two skin tissues, the expression levels of tyrosinase-related protein 1 (tyrp1), melanoma antigen recognized by T-cells 1 (mart-1) and tight junction protein 1 (tjp1) were upregulated in black-spotted skin, implying that these genes play a key role in the black coloration in $S$. argus in the eumelanin synthesis-related pathway. The expression level of tyrp 1 was upregulated in black-spotted skin compared with non-spotted skin, suggesting that tyrp 1 is a key gene that contributes to the brownish-black pigment pattern in S. argus by playing an important role in the eumelanin synthesis pathway. tyrp 1 is expressed specifically in melanocytes, and it plays a crucial role in the formation of pigment patterning by affecting melanin synthesis, stabilizing tyrosinase protein, modulating tyrosinase catalytic activity, maintaining the melanosome structure and affecting melanocyte proliferation and cell death [22-24]. Mutations of tyrp1 cannot form a black coat color in mice [25], dogs [26], cats [27], cows [28] and sheep [29]. tyrp1 plays similar roles in teleost fish species, and the 
knockout of tyrp1 gene in zebrafish and medaka hinder the formation of eumelanin [15]. Our results confirmed the role of tyrp1 gene in the skin coloration of S. argus. It has been shown that sox 10 gene can activate the mitf and raise the expression of mitf [30]. In mice, both mitf and sox10 are necessary for the expression of tyr, but in zebrafish mitf is enough for the expression of tyr [31]. In Table 2, three forms of adenylate cyclase genes (adcy5, adcy 6 and $a d c y 9$ ) were significantly expressed when comparing black-spotted skin to non-spotted skin. Adenylate cyclase activator was used to treat primary cultures of normal human melanocytes, and the activation of adenylate cyclase caused mitf to start transcription and contributed to pigmentation $[32,33]$. Our results indicated that the adenylate cyclase genes may play an important role in the process of promoting melanin synthesis for fish. tjp1, encoding a tight junction protein, was upregulated in black-spotted skin when compared with non-spotted skin, suggesting that the expression of this gene could affect the formation of pigment patterning. In a previous study, a zebrafish with a mutation functional tjp1a protein was examined [34]. It was observed that tjp1a was upregulated, as expressed in dense iridophores, but downregulated in loose-form iridophores. The mutant had a spotted pattern rather than the usual striped pattern because the dense iridophores interrupted the dark stripes, resulting in a spotted pattern. Another role of tjp1 gene in skin pigment patterning is that tjp1 protein is a scaffolding protein that links tight junction proteins to the actin cytoskeleton, and could affect the rearrangement of the cytoskeleton and the movement of the cells [35]. There were several longitudinal black stripes on both sides of the body before the formation of black spots, which may be related to the expression of tjp1 gene. We hypothesized that tjp1 might play a distinct role insofar as iridophores fix a lot of melanophores in a specific position and form black spots. However, further studies are needed.

We found that the expression levels of premelanosome protein ( $\mathrm{pmel} / \mathrm{silv}$ ), solute carrier family 7 member 2 (slc7a2) and microphthalmia-associated transcription factor (mitf) were higher in black-spotted skin. pmel is a significant eumelanin synthesis pathway gene, encoding the premelanosome protein, which is extensively expressed in pigment cells [36]. Mutations in pmel gene lead to pigmentation phenotypes in many vertebrate species. In mice, pmel is required to regulate the normal development of skin and choroidal melanocytes and retinal pigment epithelial cells [36]. In chicken, the mutation of pmel inhibited the synthesis of eumelanin in the skin and plumage [37]. Similar results were obtained in horses, in which pmel mutations reduced the concentration of black pigment in the mane and tail [38]. Our results showed that the expression level of pmel in black-spotted skin was higher than that in the caudal fin. The results further confirmed the role of pmel in the eumelanogenesis of S. argus.

In addition, compared with non-spotted skin and caudal fin, DEGs identified in black-spotted skin were significantly enriched in actin cytoskeleton and cytoskeletal proteins. Cytoskeleton proteins are essential in mechanical transduction, as the cytoskeletal network promotes the translocation of signal molecules from focal adhesion sites to the cytoplasm [39]. The pigmentation-related pathways showed significant enrichments in the black-spotted skin. We speculate that the pigmentation of black spots in fish occurred because cytoskeleton construction induced by the skeletal muscle cell movement could cause the contraction and gathering of pigment cells and promote the coloration of the skin, which explains the change in pattern from stripes to spots from the juvenile to the adult stage of the fish. We noticed that there was a stage where the caudal fin only had a yellow pattern, and we speculated that black spots gradually migrated from the skin to the caudal fin and that the black spot was separated into the current black pattern. However, further investigation is still needed to confirm our conjecture.

\section{Conclusions}

We sequenced transcripts from the skin and caudal fin of spotted scat for the first time and identified 2357 DEGs (with a fold change $\geq 2$ ). These DEG data provide a reference for screening genes for their effect on pigment patterning in S. argus. The results significantly 
enhanced our understanding of the composition of the $S$. argus skin transcriptome and the potential differences in gene expression associated with skin pigment pattern maintenance, especially the formation of black spots. Our results suggested an important role of junctions and cytoskeleton rearrangements in maintaining $S$. argus skin pigment patterns due to differential gene expression, providing a certain theoretical basis for molecular breeding in the future.

Supplementary Materials: The following are available online at https:/ / www.mdpi.com/2076-261 5/11/3/765/s1, Table S1: RNA purity of 27 samples of S. argus; Table S2: Quantitative real-time PCR (qRT-PCR) primer sequences data; Table S3: Top ten significant KEGG signaling pathways from three different groups.

Author Contributions: Conceptualization: X.L., C.T. and G.L.; data curation: X.L. and C.T.; funding acquisition: C.T. and G.L.; methodology: X.L.; project administration: G.L.; resources: Y.H. and H.S.; supervision: G.L.; visualization: writing —original draft: X.L. and C.T.; writing—review and editing: X.L., C.T. and G.L. All authors have read and agreed to the published version of the manuscript.

Funding: This study was supported by grants from the National Natural Science Foundation of China (Nos. 41706174 and Nos. 31702326), Natural Science Foundation of Guangdong Province (2017A030313101, 2018B030311050, 2019A1515010958, 2019A1515110619), Independent Project of Guangdong Province Laboratory (ZJW-2019-06), the Department of Education of Guangdong Province (2018KQNCX111 and 2019KTSCX060) and the Program for Scientific Research Start-up Funds of Guangdong Ocean University (R19026).

Institutional Review Board Statement: Not applicable.

Informed Consent Statement: Informed consent was obtained from all subjects involved in the study.

Data Availability Statement: Not applicable.

Conflicts of Interest: The authors declare no conflict of interest.

\section{References}

1. Hubbard, J.K.; Uy, J.A.; Hauber, M.E.; Hoekstra, H.E.; Safran, R.J. Vertebrate pigmentation: From underlying genes to adaptive function. Trends Genet. 2010, 26, 231-239. [CrossRef] [PubMed]

2. Protas, M.E.; Patel, N.H. Evolution of coloration patterns. Annu. Rev. Cell Dev. Biol. 2008, 24, 425-446. [CrossRef] [PubMed]

3. Rodgers, G.M.; Kelley, J.L.; Morrell, L.J. Colour change and assortment in the western rainbowfish. Anim. Behav. 2010, 79, 1025-1030. [CrossRef]

4. Jiang, Y. Comparative transcriptome analysis reveals the genetic basis of skin color variation in Common Carp. PLoS ONE 2014, 9, e108200. [CrossRef]

5. Bagnara, J.T.; Fernandez, P.J.; Fujii, R. On the blue coloration of vertebrates. Pigment Cell Res. 2007, 20, 14-26. [CrossRef]

6. Braasch, I.; Brunet, F.; Volff, J.N.; Schartl, M. Pigmentation pathway evolution after whole-genome duplication in fish. Genome Biol. Evol. 2009, 1, 479-493. [CrossRef] [PubMed]

7. Kelsh, R.N.; Brand, M.; Jiang, Y.J.; Heisenberg, C.P.; Lin, S.; Haffter, P.; Odenthal, J.; Mullins, M.C.; van Eeden, F.J.; Furutani-Seiki, M.; et al. Zebrafish pigmentation mutations and the processes of neural crest development. Development 1996, 123, 369-389. [PubMed]

8. Parichy, D.M. Evolution of danio pigment pattern development. Heredity 2006, 97, 200-210. [CrossRef] [PubMed]

9. Bagnara, T.J.; Matsumoto, J. Comparative anatomy and physiology of pigment cells in nonmammalian tissues. In The Pigmentary System: Physiology and Pathophysiology, 2nd ed.; Blackwell Publishing Ltd.: Hoboken, NJ, USA, 2007; pp. 11-59.

10. Kelsh, R.N.; Inoue, C.; Momoi, A.; Kondoh, H.; Furutani-Seiki, M.; Ozato, K.; Wakamatsu, Y. The Tomita collection of medaka pigmentation mutants as a resource for understanding neural crest cell development. Mech. Dev. 2004, 121, 841-859. [CrossRef] [PubMed]

11. Mellgren, E.M.; Johnson, S.L. The evolution of morphological complexity in zebrafish stripes. Trends Genet. 2002, 18, 128-134. [CrossRef]

12. Braasch, I.; Schartl, M.; Volff, J.N. Evolution of pigment synthesis pathways by gene and genome duplication in fish. BMC Evol. Biol. 2007, 7, 74. [CrossRef]

13. Li, S.; Wang, C.; Yu, W.; Zhao, S.; Gong, Y. Identification of genes related to white and black plumage formation by RNA-Seq from white and black feather bulbs in ducks. PLoS ONE 2012, 7, e36592. [CrossRef] [PubMed]

14. Logan, D.W.; Burn, S.F.; Jackson, I.J. Regulation of pigmentation in zebrafish melanophores. Pigment Cell Res. 2006, 19, $206-213$. [CrossRef] [PubMed] 
15. Braasch, I.; Liedtke, D.; Volff, J.N.; Schartl, M. Pigmentary function and evolution of tyrp1 gene duplicates in fish. Pigment Cell Melanoma Res. 2009, 22, 839-850. [CrossRef] [PubMed]

16. Altschmied, J.; Delfgaauw, J.; Wilde, B.; Wilde, B.; Duschl, J.; Bouneau, L.; Bouneau, L.; Schartl, M. Subfunctionalization of duplicate mitf genes associated with differential degeneration of alternative exons in fish. Genetics 2002, 161, 259-267. [PubMed]

17. Trapnell, C.; Williams, B.A.; Pertea, G.; Mortazavi, A.; Kwan, G.; van Baren, M.J.; Salzberg, S.L.; Wold, B.J.; Pachter, L. Transcript assembly and quantification by RNA-Seq reveals unannotated transcripts and isoform switching during cell differentiation. Nat. Biotechnol. 2010, 28, 511. [CrossRef]

18. Varet, H.; Brillet-Guéguen, L.; Coppée, J.Y.; Dillies, M.A. SAR Tools: A DESeq2- and edge R-based R pipeline for comprehensive differential analysis of RNA-Seq data. PLoS ONE 2016, 11, e0157022. [CrossRef]

19. Anders, S.; Huber, W. Differential expression analysis for sequence count data. Genome Biol. 2010, 11, R106. [CrossRef]

20. Livak, K.J.; Schmittgen, T.D. Analysis of relative gene expression data using real-time quantitative PCR and the 2- $\Delta \Delta C T$ method. Methods 2001, 25, 402-408. [CrossRef]

21. Djurdjevič, I.; Kreft, M.E.; Sušnik, B.S. Comparison of pigment cell ultrastructure and organisation in the dermis of marble trout and brown trout, and first description of erythrophore ultrastructure in salmonids. J. Anat. 2015, 227, 583-595. [CrossRef]

22. Rad, H.H.; Yamashita, T.; Jin, H.Y.; Hirosaki, K.; Wakamatsu, K.; Ito, S.; Jimbow, K. Tyrosinase-related proteins suppress tyrosinase-mediated cell death of melanocytes and melanoma cells. Exp. Cell Res. 2004, 298, 317-328. [CrossRef] [PubMed]

23. Sarangarajan, R.; Boissy, R.E. Tyrp1 and oculocutaneous albinism type 3. Pigment Cell Res. 2001, 14, 437-444. [CrossRef] [PubMed]

24. Kobayashi, T.; Hearing, V.J. Direct interaction of tyrosinase with Tyrp1 to form heterodimeric complexes in vivo. J. Cell Sci. 2007, 120, 4261-4268. [CrossRef]

25. Shibahara, S.; Tomita, Y.; Yoshizawa, M.; Shibata, K.; Tagami, H. Identification of mutations in the pigment cell-specific gene located at the brown locus in mouse. Pigment Cell Res. Suppl. 2010, 3, 90-95. [CrossRef] [PubMed]

26. Schmutz, S.M.; Berryere, T.G.; Goldfinch, A.D. TYRP1 and MC1R genotypes and their effects on coat color in dogs. Mamm. Genome 2002, 13, 380-387. [CrossRef]

27. Schmidt-Kuntzel, A.; Eizirik, E.; O’Brien, S.J.; Menotti-Raymond, M. Tyrosinase and tyrosinase related protein 1 alleles specify domestic cat coat color phenotypes of the albino and brown loci. J. Hered. 2005, 96, 289-301. [CrossRef]

28. Berryere, T.G.; Schmutz, S.M.; Schimpf, R.J.; Cowan, C.M.; Potter, J. TYRP1 is associated with dun coat colour in Dexter cattle or how now brown cow? Anim. Genet. 2003, 34, 169-175. [CrossRef]

29. Gratten, J.; Beraldi, D.; Lowder, B.V.; McRae, A.F.; Visscher, P.M. Compelling evidence that a single nucleotide substitution in TYRP1 is responsible for coat-colour polymorphism in a free-living population of Soay sheep. Proc. Biol. Sci. 2007, 274, 619-626. [CrossRef]

30. Mollaaghababa, R.; Pavan, W.J. The importance of having your SOX on: Role of SOX10 in the development of neural crest-derived melanocytes and glia. Oncogene 2003, 22, 3024-3034. [CrossRef]

31. Hou, L.; Arnheiter, H.; Pavan, W.J. Interspecies difference in the regulation of melanocyte development by SOX10 and MITF. Proc. Natl. Acad. Sci. USA 2006, 103, 9081-9085. [CrossRef]

32. Newton, R.A.; Cook, A.L.; Roberts, D.W.; Leonard, J.H.; Sturm, R.A. Post-transcriptional regulation of melanin biosynthetic enzymes by cAMP and resveratrol in human melanocytes. J. Investig. Dermatol. 2007, 127, 2216-2227. [CrossRef]

33. Oren, M.; Bartek, J. The sunny side of p53. Cell 2007, 128, 826-828. [CrossRef]

34. Fadeev, A.; Krauss, J.; Frohnhöfer, H.G.; Irion, U.; Nüsslein-Volhard, C. Tight junction protein 1a regulates pigment cell organisation during zebrafish colour patterning. eLife 2015, 4, 1-25. [CrossRef] [PubMed]

35. Miyoshi, J.; Takai, Y. Structural and functional associations of apical junctions with cytoskeleton. Biochim. Biophys. Acta Biomembr. 2008, 1778, 670-691. [CrossRef] [PubMed]

36. Hellstrom, A.R.; Watt, B.; Fard, S.S.; Tenza, D.; Mannstrom, P.; Narfstrōm, K.; Ekesten, B.; Ito, S.; Wakamatsu, K.; Larsson, J.; et al. Inactivation of Pmel alters melanosome shape but has only a subtle effect on visible pigmentation. PLoS Genet. 2011, 7, e1002285. [CrossRef] [PubMed]

37. Karlsson, A.C.; Kerje, S.; Hallbook, F.; Jensen, P. The dominant white mutation in the PMEL17 gene does not cause visual impairment in chickens. Vet. Ophthalmol. 2009, 12, 292-298. [CrossRef]

38. Brunberg, E.; Andersson, L.; Cothran, G.; Sandberg, K.; Mikko, S.; Lindgren, G. A missense mutation in PMEL17 is associated with the Silver coat color in the horse. BMC Genet. 2006, 7, 46. [CrossRef] [PubMed]

39. Shyy, J.Y.; Chien, S. Role of integrins in endothelial mechanosensing of shear stress. Circ. Res. 2002, 91, 769-775. [CrossRef] [PubMed] 